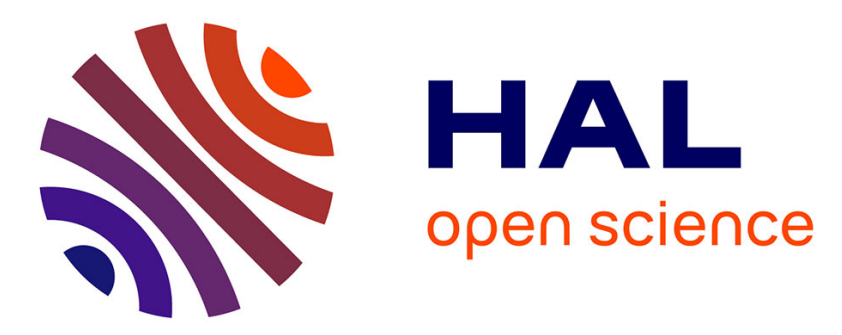

\title{
C2 interpolation of spatial data subject to arc-length constraints using Pythagorean-hodograph quintic splines
}

\author{
Mathieu Huard, Rida Farouki, Nathalie Sprinsky, Luc Biard
}

\section{To cite this version:}

Mathieu Huard, Rida Farouki, Nathalie Sprinsky, Luc Biard. C2 interpolation of spatial data subject to arc-length constraints using Pythagorean-hodograph quintic splines. Graphical Models, 2014, 76

(1), pp.30-42. 10.1016/j.gmod.2013.10.005 . hal-00983444

\section{HAL Id: hal-00983444 \\ https://hal.science/hal-00983444}

Submitted on 25 Apr 2014

HAL is a multi-disciplinary open access archive for the deposit and dissemination of scientific research documents, whether they are published or not. The documents may come from teaching and research institutions in France or abroad, or from public or private research centers.
L'archive ouverte pluridisciplinaire HAL, est destinée au dépôt et à la diffusion de documents scientifiques de niveau recherche, publiés ou non, émanant des établissements d'enseignement et de recherche français ou étrangers, des laboratoires publics ou privés. 


\title{
$C^{2}$ interpolation of spatial data subject to arc-length constraints using Pythagorean-hodograph quintic splines
}

\author{
Mathieu Huard, ${ }^{1}$ Rida T. Farouki, ${ }^{2}$ Nathalie Sprynski, ${ }^{1}$ Luc Biard ${ }^{3}$ \\ ${ }^{1}$ CEA-LETI, MINATEC Campus, Grenoble, France \\ ${ }^{2}$ Department of Mechanical and Aerospace Engineering, University of California, Davis \\ ${ }^{3}$ Laboratoire Jean Kuntzmann, Université Joseph Fourier, Grenoble, France
}

\begin{abstract}
In order to reconstruct spatial curves from discrete electronic sensor data, two alternative $C^{2}$ Pythagorean-hodograph (PH) quintic spline formulations are proposed, interpolating given spatial data subject to prescribed constraints on the arc length of each spline segment. The first approach is concerned with the interpolation of a sequence of points, while the second addresses the interpolation of derivatives only (without spatial localization). The special structure of $\mathrm{PH}$ curves allows the arc-length conditions to be expressed as algebraic constraints on the curve coefficients. The $C^{2} \mathrm{PH}$ quintic splines are thus defined through minimization of a quadratic function subject to quadratic constraints, and a close starting approximation to the desired solution is identified in order to facilitate efficient construction by iterative methods. The $C^{2}$ $\mathrm{PH}$ spline constructions are illustrated by several computed examples.
\end{abstract}

e-mail: mathieu.huard@cea.fr, farouki@ucdavis.edu, nathalie.sprynski@cea.fr, Luc.Biard@imag.fr

corresponding author: R. T. Farouki (e-mail: farouki@ucdavis.edu, phone: 530-752-1779, fax: 530-752-4158)

\section{Introduction}

The Leti ${ }^{1}$ department of the CEA ${ }^{2}$ in Grenoble, France has recently developed the Morphosense, a flexible ribbon-like device instrumented with micro-accelerometers and micro-magnetometers according to a known distribution along its length [3]. Each sensor knot provides measurements that can be used to completely determine the spatial orientation of the knot. From this orientation data, and the known distances between consecutive sensors, it is desired to reconstruct the geometrical shape assumed by the Morphosense when placed in contact with a physical object (see Figure 1).

It should be emphasized that the Morphosense shape recovery is not simply a classical Hermite or spline interpolation problem, since the absolute (or even relative) spatial locations of the sensor knots are not known. Instead, only the curvilinear distances between successive sensors are known. Thus, the shape recovery problem amounts to constructing a spline curve that interpolates a given

\footnotetext{
${ }^{1}$ Laboratoire d'électronique des technologies de l'information.

${ }^{2}$ Commissariat à l'Energie Atomique et aux Energies Alternatives.
} 


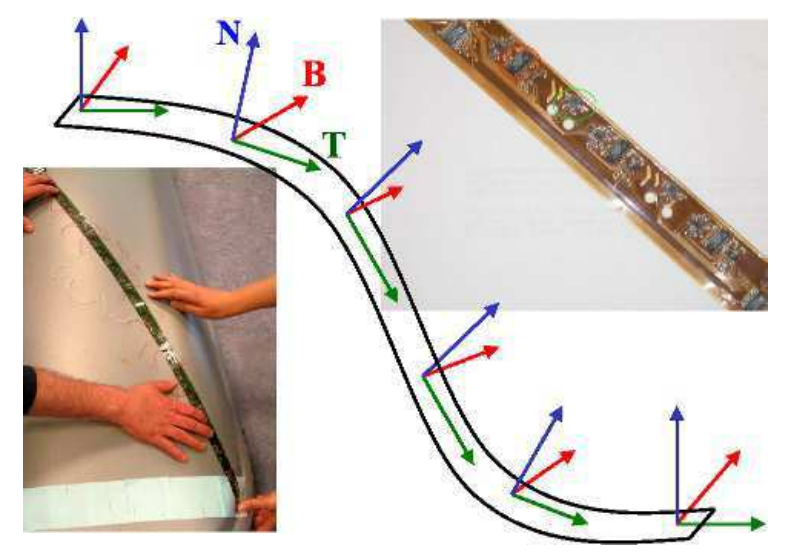

Figure 1: Discrete geometric data furnished by the Morphosense ribbon for shape reconstruction.

sequence of Serret-Frenet frames, with precribed arc lengths for each segment between such frames. This problem has been previously studied $[11,12,14]$ assuming an arc-length parameterization, which allows control over the curve segment lengths between sensors. The proposed methods aim to interpolate the tangent indicatrix of the curve on the unit sphere. Since they rely on numerical integration, however, such methods do not yield analytic definitions of the reconstructed curve.

A new global approach to the shape reconstruction is presented herein, based on the polynomial Pythagorean-hodograph (PH) curves [5]. The proposed solutions are spatial $C^{2} \mathrm{PH}$ quintic splines, with segments that match the arc-length constraints. Specifically, two spatial $C^{2} \mathrm{PH}$ quintic spline interpolation schemes, subject to segment arc-length constraints, are developed. The first scheme interpolates a given sequence of points in $\mathbb{R}^{3}$, while the second interpolates given derivative vectors (providing an approximate solution to the Morphosense shape reconstruction problem).

The distinctive feature of polynomial PH curves is that their parametric speed, i.e., the derivative of arc length with respect to the curve parameter) is a polynomial - rather than the square root of a polynomial - in the curve parameter. This special algebraic structure of $\mathrm{PH}$ curves offers many useful computational advantages over "ordinary" polynomial parametric curves. For example, the $\mathrm{PH}$ curves admit rational offsets and exact arc-length computation, and are well-suited to real-time CNC interpolator algorithms, used to drive computer-controlled machines along curved paths with a prescribed speed variation. A comprehensive treatment may be found in [5].

The construction of planar $C^{2} \mathrm{PH}$ spline interpolants involves the solution of a system of $N$ quadratic equations in $N$ complex unknowns $[1,8,13]$. In the construction of spatial $C^{2} \mathrm{PH}$ splines, however, there are more freedoms ${ }^{3}$ than are required to satisfy the interpolation conditions $[9,10]$. A method was presented in [10] to fix the residual freedoms by optimizing a shape measure for the interpolant. In the present context, the residual freedoms will be exploited to achieve satisfaction of the prescribed arc-length constraints for successive spline segments, a physically and geometrically important aspect of the Morphosense shape reconstruction problem.

The remainder of this paper is organized as follows. Section 2 first presents some preliminaries concerning the definition and properties of spatial PH curves. The system of equations that defines a spatial $C^{2} \mathrm{PH}$ quintic spline is then introduced in Section 3. The interpolation of a set of points

${ }^{3}$ These extra freedoms are necessary to ensure a rotation-invariant representation $[6,7]$. 
under segment arc-length constraints is discussed in Section 4, and the interpolation of derivatives under segment arc-length constraints is addressed in Section 5. The methods are illustrated using computed examples in both cases. Finally, Section 6 summarizes the capabilities and limitations of these methods, and briefly discusses possible enhancements and extensions.

\section{Spatial Pythagorean-hodograph curves}

A polynomial $\mathrm{PH}$ curve $\mathbf{r}(t)=(x(t), y(t), z(t))$ in $\mathbb{R}^{3}$ is characterized by the fact that its hodograph or derivative $\mathbf{r}^{\prime}(t)=\left(x^{\prime}(t), y^{\prime}(t), z^{\prime}(t)\right)$ satisfies the Pythagorean relation

$$
x^{\prime 2}(t)^{2}+y^{\prime 2}(t)+z^{\prime 2}(t)=\sigma^{2}(t)
$$

for some polynomial $\sigma(t)$. The polynomial $\sigma(t)=\left|\mathbf{r}^{\prime}(t)\right|$ is the parametric speed of the curve - i.e., the rate of change $\mathrm{d} s / \mathrm{d} t$ of its arc length $s$ with respect to the curve parameter $t$. The Pythagorean condition (1) is satisfied if and only if the hodograph components can be expressed in terms of four polynomials $u(t), v(t), p(t), q(t)$ in the form

$$
\begin{aligned}
x^{\prime}(t) & :=u^{2}(t)+v^{2}(t)-p^{2}(t)-q^{2}(t), \\
y^{\prime}(t) & :=2[u(t) q(t)+v(t) p(t)], \\
z^{\prime}(t) & :=2[v(t) q(t)-u(t) p(t)],
\end{aligned}
$$

and the parametric speed is then

$$
\sigma(t):=u^{2}(t)+v^{2}(t)+p^{2}(t)+q^{2}(t) .
$$

The quaternion and Hopf map forms [2] are two alternative (equivalent) formulations of spatial PH curves that embody the structure (1)-(3). The Hopf map form will be adopted here. This generates the form (2) from a pair of complex ${ }^{4}$ polynomials

$$
\boldsymbol{\alpha}(t):=u(t)+\mathrm{i} v(t), \quad \boldsymbol{\beta}(t):=q(t)+\mathrm{i} p(t)
$$

through the expression

$$
\mathbf{r}^{\prime}(t):=\left(|\boldsymbol{\alpha}(t)|^{2}-|\boldsymbol{\beta}(t)|^{2}, 2 \operatorname{Re}(\boldsymbol{\alpha}(t) \overline{\boldsymbol{\beta}}(t)), 2 \operatorname{Im}(\boldsymbol{\alpha}(t) \overline{\boldsymbol{\beta}}(t))\right.
$$

(where $\overline{\mathbf{z}}$ denotes the complex conjugate of $\mathbf{z}$ ), and the parametric speed is then

$$
\sigma(t):=|\boldsymbol{\alpha}(t)|^{2}+|\boldsymbol{\beta}(t)|^{2} .
$$

The simplest non-trivial spatial PH curves are the cubics, generated by choosing linear complex polynomials for (4), substituting in (5), and integrating. Since the spatial PH cubics have insufficient shape flexibility for $C^{2}$ spline interpolation, however, we employ PH quintics. These are defined in terms of two quadratic complex polynomials, specified in Bernstein form as

$$
\begin{aligned}
\boldsymbol{\alpha}(t) & :=\boldsymbol{\alpha}_{0}(1-t)^{2}+\boldsymbol{\alpha}_{1} 2(1-t) t+\boldsymbol{\alpha}_{2} t^{2}, \\
\boldsymbol{\beta}(t) & :=\boldsymbol{\beta}_{0}(1-t)^{2}+\boldsymbol{\beta}_{1} 2(1-t) t+\boldsymbol{\beta}_{2} t^{2} .
\end{aligned}
$$

\footnotetext{
${ }^{4}$ Bold characters will be used to denote both complex numbers and points/vectors in $\mathbb{R}^{3}$ — the meaning should be clear from the context.
} 
Writing a point $(x, y, z) \in \mathbb{R}^{3}$ in the form $(x, y+\mathrm{i} z)$, the control points $\mathbf{q}_{k}=\left(x_{k}, y_{k}+\mathrm{i} z_{k}\right)$ for the Bézier form

$$
\mathbf{r}(t):=\sum_{k=0}^{5} \mathbf{q}_{k}\left(\begin{array}{l}
5 \\
k
\end{array}\right)(1-t)^{5-k} t^{k}
$$

of the PH quintic generated by substituting $\boldsymbol{\alpha}(t), \boldsymbol{\beta}(t)$ into (5) and integrating can be written as

$$
\begin{aligned}
\mathbf{q}_{1} & :=\mathbf{q}_{0}+\frac{1}{5}\left(\left|\boldsymbol{\alpha}_{0}\right|^{2}-\left|\boldsymbol{\beta}_{0}\right|^{2}, 2 \boldsymbol{\alpha}_{0} \boldsymbol{\beta}_{0}\right) \\
\mathbf{q}_{2} & :=\mathbf{q}_{1}+\frac{1}{5}\left(\operatorname{Re}\left(\boldsymbol{\alpha}_{0} \overline{\boldsymbol{\alpha}}_{1}-\boldsymbol{\beta}_{0} \overline{\boldsymbol{\beta}}_{1}\right), \boldsymbol{\alpha}_{0} \overline{\boldsymbol{\beta}}_{1}+\boldsymbol{\alpha}_{1} \overline{\boldsymbol{\beta}}_{0}\right), \\
\mathbf{q}_{3} & :=\mathbf{q}_{2}+\frac{1}{15}\left(2\left(\left|\boldsymbol{\alpha}_{1}\right|^{2}-\left|\boldsymbol{\beta}_{1}\right|^{2}\right)+\operatorname{Re}\left(\boldsymbol{\alpha}_{2} \overline{\boldsymbol{\alpha}}_{0}-\boldsymbol{\beta}_{2} \overline{\boldsymbol{\beta}}_{0}\right), 4 \boldsymbol{\alpha}_{1} \overline{\boldsymbol{\beta}}_{1}+\boldsymbol{\alpha}_{0} \overline{\boldsymbol{\beta}}_{2}+\boldsymbol{\alpha}_{2} \overline{\boldsymbol{\beta}}_{0}\right), \\
\mathbf{q}_{4} & :=\mathbf{q}_{3}+\frac{1}{5}\left(\operatorname{Re}\left(\boldsymbol{\alpha}_{1} \overline{\boldsymbol{\alpha}}_{2}-\boldsymbol{\beta}_{1} \overline{\boldsymbol{\beta}}_{2}\right), \boldsymbol{\alpha}_{1} \overline{\boldsymbol{\beta}}_{2}+\boldsymbol{\alpha}_{2} \overline{\boldsymbol{\beta}}_{1}\right), \\
\mathbf{q}_{5} & :=\mathbf{q}_{4}+\frac{1}{5}\left(\left|\boldsymbol{\alpha}_{2}\right|^{2}-\left|\boldsymbol{\beta}_{2}\right|^{2}, 2 \boldsymbol{\alpha}_{2} \boldsymbol{\beta}_{2}\right)
\end{aligned}
$$

where $\mathbf{q}_{0}=\left(x_{0}, y_{0}+\mathrm{i} z_{0}\right)$ is a freely-chosen integration constant.

Remark 1. If $\boldsymbol{\alpha}_{0}-2 \boldsymbol{\alpha}_{1}+\boldsymbol{\alpha}_{2}=\boldsymbol{\beta}_{0}-2 \boldsymbol{\beta}_{1}+\boldsymbol{\beta}_{2}=0$, then $\boldsymbol{\alpha}(t)$ and $\boldsymbol{\beta}(t)$ are actually degree-elevated linear polynomials, and the resulting $\mathrm{PH}$ curve is a cubic rather than a quintic.

\section{$3 \quad C^{2} \mathbf{P H}$ quintic spline equations}

For brevity, we restrict our attention here to uniformly-parameterized splines - i.e., the parameter domain for each spline segment $\mathbf{r}_{i}(t)$ is $t \in[0,1]$. This is appropriate to the Morphosense shape reconstruction problem, since the sensors typically have a uniform distribution along its length. Details of the generalization to non-uniform parameterizations may be found in [9].

Given a set of complex coefficients $\left(\mathbf{a}_{i}, \mathbf{b}_{i}\right)$ for $i=0, \ldots, N+1$, consider the $N$ quintic PH curves $\mathbf{r}_{i}(t), t \in[0,1]$ generated for $i=1, \ldots, N$ by the complex quadratic polynomials

$$
\begin{aligned}
\boldsymbol{\alpha}_{i}(t) & :=\frac{1}{2}\left(\mathbf{a}_{i-1}+\mathbf{a}_{i}\right)(1-t)^{2}+\mathbf{a}_{i} 2(1-t) t+\frac{1}{2}\left(\mathbf{a}_{i}+\mathbf{a}_{i+1}\right) t^{2}, \\
\boldsymbol{\beta}_{i}(t) & :=\frac{1}{2}\left(\mathbf{b}_{i-1}+\mathbf{b}_{i}\right)(1-t)^{2}+\mathbf{b}_{i} 2(1-t) t+\frac{1}{2}\left(\mathbf{b}_{i}+\mathbf{b}_{i+1}\right) t^{2} .
\end{aligned}
$$

Adoption of this form automatically guarantees continuity of first and second derivatives between consecutive PH spline segments $\mathbf{r}_{i}(t)$ and $\mathbf{r}_{i+1}(t)$. Specifically, one can verify [10] that

$$
\begin{aligned}
\mathbf{r}_{i}^{\prime}(1) & =\mathbf{r}_{i+1}^{\prime}(0)=\left(\frac{1}{4}\left(\left|\mathbf{a}_{i}+\mathbf{a}_{i+1}\right|^{2}-\left|\mathbf{b}_{i}+\mathbf{b}_{i+1}\right|^{2}\right), \frac{1}{2}\left(\mathbf{a}_{i}+\mathbf{a}_{i+1}\right)\left(\overline{\mathbf{b}}_{i}+\overline{\mathbf{b}}_{i+1}\right)\right), \\
\mathbf{r}_{i}^{\prime \prime}(1) & =\mathbf{r}_{i+1}^{\prime \prime}(0)=\left(\left|\mathbf{a}_{i+1}\right|^{2}-\left|\mathbf{a}_{i}\right|^{2}+\left|\mathbf{b}_{i+1}\right|^{2}-\left|\mathbf{b}_{i}\right|^{2}, 2\left(\mathbf{a}_{i+1} \overline{\mathbf{b}}_{i+1}-\mathbf{a}_{i} \overline{\mathbf{b}}_{i}\right)\right) .
\end{aligned}
$$

Note that, although $C^{1}$ and $C^{2}$ continuity is thereby ensured, one must still enforce $C^{0}$ continuity. Integration of the hodograph $\mathbf{r}_{i}^{\prime}(t)$ allows the initial point $\mathbf{r}_{i}(0)$ of each segment to be freely chosen, but satisfaction of the $C^{0}$ condition $\mathbf{r}_{i}(1)=\mathbf{r}_{i+1}(0)$ with the succeeding segment must be achieved by means of an appropriate scheme (see Sections 4 and 5 below).

From (8), one can verify that the total arc length

$$
l_{i}:=\int_{0}^{1}\left|\boldsymbol{\alpha}_{i}(t)\right|^{2}+\left|\boldsymbol{\beta}_{i}(t)\right|^{2} \mathrm{~d} t
$$


of the spline segment $\mathbf{r}_{i}(t)$ is defined in terms of the complex coefficients $\mathbf{a}_{i}, \mathbf{b}_{i}$ by the expression

$$
\begin{aligned}
120 l_{i} & =6\left(\left|\mathbf{a}_{i-1}\right|^{2}+\left|\mathbf{b}_{i-1}\right|^{2}\right)+54\left(\left|\mathbf{a}_{i}\right|^{2}+\left|\mathbf{b}_{i}\right|^{2}\right)+6\left(\left|\mathbf{a}_{i+1}\right|^{2}+\left|\mathbf{b}_{i+1}\right|^{2}\right) \\
& +13\left(\mathbf{a}_{i-1} \overline{\mathbf{a}}_{i}+\mathbf{b}_{i-1} \overline{\mathbf{b}}_{i}+\overline{\mathbf{a}}_{i-1} \mathbf{a}_{i}+\overline{\mathbf{b}}_{i-1} \mathbf{b}_{i}+\mathbf{a}_{i} \overline{\mathbf{a}}_{i+1}+\mathbf{b}_{i} \overline{\mathbf{b}}_{i+1}+\overline{\mathbf{a}}_{i} \mathbf{a}_{i+1}+\overline{\mathbf{b}}_{i} \mathbf{b}_{i+1}\right) \\
& +\mathbf{a}_{i-1} \overline{\mathbf{a}}_{i+1}+\mathbf{b}_{i-1} \overline{\mathbf{b}}_{i+1}+\overline{\mathbf{a}}_{i-1} \mathbf{a}_{i+1}+\overline{\mathbf{b}}_{i-1} \mathbf{b}_{i+1} .
\end{aligned}
$$

Note that, although $l_{i}$ is expressed in terms of complex numbers, it has a real value.

\section{Interpolation of points under length constraints}

Consider first the problem of interpolating a sequence of points by a spatial $C^{2} \mathrm{PH}$ quintic spline, with prescribed arc lengths for each spline segment. Specifically, given points $\mathbf{p}_{0}, \ldots, \mathbf{p}_{N} \in \mathbb{R}^{3}$ and segment arc lengths $l_{1}, \ldots, l_{N}$ the problem is to identify a set of PH quintic segments $\mathbf{r}_{1}(t), \ldots, \mathbf{r}_{N}(t)$ defined on $t \in[0,1]$ such that

$$
\mathbf{r}_{i}(1)=\mathbf{r}_{i+1}(0)=\mathbf{p}_{i}, \quad i=1, \ldots, N-1 \quad \text { and } \quad \int_{0}^{1}\left|\mathbf{r}_{i}^{\prime}(t)\right| \mathrm{d} t=l_{i}, \quad i=1, \ldots, N
$$

with $\mathbf{r}_{1}(0)=\mathbf{p}_{0}$ and $\mathbf{r}_{N}(1)=\mathbf{p}_{N}$. By using the polynomials (8), the first and second derivative continuity conditions (9) are automatically satisfied.

\subsection{Interpolation of points}

Each segment $\mathbf{r}_{i}(t)$ for $i=1, \ldots, N$ is required to satisfy $\mathbf{r}_{i}(0)=\mathbf{p}_{i-1}$ and $\mathbf{r}_{i}(1)=\mathbf{p}_{i}$. On integrating the hodograph $\mathbf{r}_{i}^{\prime}(t)$, one can choose $\mathbf{r}_{i}(0)=\mathbf{p}_{i-1}$ as the integration constant. Achieving $\mathbf{r}_{i}(1)=\mathbf{p}_{i}$ then corresponds to satisfying the equation

$$
\int_{0}^{1} \mathbf{r}_{i}^{\prime}(t) \mathrm{d} t=\mathbf{p}_{i}-\mathbf{p}_{i-1}:=\Delta \mathbf{p}_{i}
$$

for $i=1, . ., N$. Writing $\Delta \mathbf{p}_{i}=\left(\Delta x_{i}, \Delta y_{i}+\mathrm{i} \Delta z_{i}\right)$ and using (5), this equation is equivalent to

$$
\int_{0}^{1}\left|\boldsymbol{\alpha}_{i}(t)\right|^{2}-\left|\boldsymbol{\beta}_{i}(t)\right|^{2} \mathrm{~d} t=\Delta x_{i}, \quad \int_{0}^{1} 2 \boldsymbol{\alpha}_{i}(t) \overline{\boldsymbol{\beta}}_{i}(t) \mathrm{d} t=\Delta y_{i}+\mathrm{i} \Delta z_{i},
$$

which can be expressed in terms of the complex numbers $\mathbf{a}_{i}, \mathbf{b}_{i}$ as

$$
\begin{aligned}
& 6\left(\left|\mathbf{a}_{i-1}\right|^{2}-\left|\mathbf{b}_{i-1}\right|^{2}\right)+54\left(\left|\mathbf{a}_{i}\right|^{2}-\left|\mathbf{b}_{i}\right|^{2}\right)+6\left(\left|\mathbf{a}_{i+1}\right|^{2}-\left|\mathbf{b}_{i+1}\right|^{2}\right) \\
& +13\left(\mathbf{a}_{i-1} \overline{\mathbf{a}}_{i}-\mathbf{b}_{i-1} \overline{\mathbf{b}}_{i}+\overline{\mathbf{a}}_{i-1} \mathbf{a}_{i}-\overline{\mathbf{b}}_{i-1} \mathbf{b}_{i}+\mathbf{a}_{i} \overline{\mathbf{a}}_{i+1}-\mathbf{b}_{i} \overline{\mathbf{b}}_{i+1}+\overline{\mathbf{a}}_{i} \mathbf{a}_{i+1}-\overline{\mathbf{b}}_{i} \mathbf{b}_{i+1}\right) \\
& +\mathbf{a}_{i-1} \overline{\mathbf{a}}_{i+1}-\mathbf{b}_{i-1} \overline{\mathbf{b}}_{i+1}+\overline{\mathbf{a}}_{i-1} \mathbf{a}_{i+1}-\overline{\mathbf{b}}_{i-1} \mathbf{b}_{i+1}=120 \Delta x_{i}, \\
& 6 \mathbf{a}_{i-1} \overline{\mathbf{b}}_{i-1}+54 \mathbf{a}_{i} \overline{\mathbf{b}}_{i}+6 \mathbf{a}_{i+1} \overline{\mathbf{b}}_{i+1} \\
& +13\left(\mathbf{a}_{i-1} \overline{\mathbf{b}}_{i}+\mathbf{a}_{i+1} \overline{\mathbf{b}}_{i}+\mathbf{a}_{i} \overline{\mathbf{b}}_{i-1}+\mathbf{a}_{i} \overline{\mathbf{b}}_{i+1}\right) \\
& +\mathbf{a}_{i-1} \overline{\mathbf{b}}_{i+1}+\mathbf{a}_{i+1} \overline{\mathbf{b}}_{i-1}=60\left(\Delta y_{i}+\mathrm{i} \Delta z_{i}\right),
\end{aligned}
$$

for $i=1, \ldots, N$. Thus, since equations (14) are real and equations (15) are complex, interpolation of the points $\mathbf{p}_{0}, \ldots, \mathbf{p}_{N}$ is achieved through the satisfaction $3 N$ scalar conditions on the complex unknowns $\mathbf{a}_{0}, \ldots, \mathbf{a}_{N+1}$ and $\mathbf{b}_{0}, \ldots, \mathbf{b}_{N+1}$. 


\subsection{Satisfaction of length constraints}

From (11), satisfaction of the arc length constraints yields the equations

$$
\begin{aligned}
& 6\left(\left|\mathbf{a}_{i-1}\right|^{2}+\left|\mathbf{b}_{i-1}\right|^{2}\right)+54\left(\left|\mathbf{a}_{i}\right|^{2}+\left|\mathbf{b}_{i}\right|^{2}\right)+6\left(\left|\mathbf{a}_{i+1}\right|^{2}+\left|\mathbf{b}_{i+1}\right|^{2}\right) \\
& +13\left(\mathbf{a}_{i-1} \overline{\mathbf{a}}_{i}+\mathbf{b}_{i-1} \overline{\mathbf{b}}_{i}+\overline{\mathbf{a}}_{i-1} \mathbf{a}_{i}+\overline{\mathbf{b}}_{i-1} \mathbf{b}_{i}+\mathbf{a}_{i} \overline{\mathbf{a}}_{i+1}+\mathbf{b}_{i} \overline{\mathbf{b}}_{i+1}+\overline{\mathbf{a}}_{i} \mathbf{a}_{i+1}+\overline{\mathbf{b}}_{i} \mathbf{b}_{i+1}\right) \\
& +\mathbf{a}_{i-1} \overline{\mathbf{a}}_{i+1}+\mathbf{b}_{i-1} \overline{\mathbf{b}}_{i+1}+\overline{\mathbf{a}}_{i-1} \mathbf{a}_{i+1}+\overline{\mathbf{b}}_{i-1} \mathbf{b}_{i+1}=120 l_{i}
\end{aligned}
$$

for $i=1, \ldots, N$. Comparing equation (10) and the first equation in (13), it is apparent that (16) differs from (14) only in the replacement of all minus signs with plus signs on the left-hand side. The real equations (14) and (16) can thus be simplified by replacing them with one-half of their sum and their difference, namely

$$
\begin{aligned}
& \quad 6\left|\mathbf{a}_{i-1}\right|^{2}+54\left|\mathbf{a}_{i}\right|^{2}+6\left|\mathbf{a}_{i+1}\right|^{2} \\
& +13\left(\mathbf{a}_{i-1} \overline{\mathbf{a}}_{i}+\overline{\mathbf{a}}_{i-1} \mathbf{a}_{i}+\mathbf{a}_{i} \overline{\mathbf{a}}_{i+1}+\overline{\mathbf{a}}_{i} \mathbf{a}_{i+1}\right) \\
& +\mathbf{a}_{i-1} \overline{\mathbf{a}}_{i+1}+\overline{\mathbf{a}}_{i-1} \mathbf{a}_{i+1}=60\left(l_{i}+\Delta x_{i}\right), \\
& \\
& \quad 6\left|\mathbf{b}_{i-1}\right|^{2}+54\left|\mathbf{b}_{i}\right|^{2}+6\left|\mathbf{b}_{i+1}\right|^{2} \\
& +13\left(\mathbf{b}_{i-1} \overline{\mathbf{b}}_{i}+\overline{\mathbf{b}}_{i-1} \mathbf{b}_{i}+\mathbf{b}_{i} \overline{\mathbf{b}}_{i+1}+\overline{\mathbf{b}}_{i} \mathbf{b}_{i+1}\right) \\
& +\mathbf{b}_{i-1} \overline{\mathbf{b}}_{i+1}+\overline{\mathbf{b}}_{i-1} \mathbf{b}_{i+1}=60\left(l_{i}-\Delta x_{i}\right) .
\end{aligned}
$$

Interpolating the points $\mathbf{p}_{0}, \ldots, \mathbf{p}_{N}$ with prescribed segment arc lengths $l_{1}, \ldots, l_{N}$ thus amounts to solving the system defined by the $N$ complex equations (15) and $2 N$ real equations (17)-(18) for $i=1, \ldots, N$. Altogether, these amount to imposing $4 N$ scalar conditions on the $4 N+8$ scalar degrees of freedom embodied in the complex values $\mathbf{a}_{0}, \ldots, \mathbf{a}_{N+1}$ and $\mathbf{b}_{0}, \ldots, \mathbf{b}_{N+1}$.

\subsection{Shape optimization procedure}

In classical $C^{2}$ cubic spline interpolation problems, end conditions must be imposed on the first and last segment, in order to match the number of equations and free unknowns. The commonly-used end conditions are (i) quadratic end spans, involving degree reduction of $\mathbf{r}_{1}(t)$ and $\mathbf{r}_{N}(t)$; (ii) the "not-a-knot" condition, in which $\mathbf{r}_{1}(t), \mathbf{r}_{2}(t)$ and $\mathbf{r}_{N-1}(t), \mathbf{r}_{N}(t)$ meet with $C^{3}$ continuity; and (iii) periodic end conditions, appropriate to smooth closed curves [4].

Since the present focus is on open curves, analogs to end conditions (i) or (ii) must be considered for $C^{2} \mathrm{PH}$ quintic splines. The "not-a-knot" condition is difficult to formulate with $\mathrm{PH}$ curves, but a degree reduction of $\mathbf{r}_{1}(t)$ and $\mathbf{r}_{N}(t)$ from quintic to cubic can be easily implemented. It occurs when $\boldsymbol{\alpha}_{1}(t), \boldsymbol{\beta}_{1}(t)$ and $\boldsymbol{\alpha}_{N}(t), \boldsymbol{\beta}_{N}(t)$ are actually degree-elevated linear (rather than true quadratic) polynomials. By Remark 1, this occurs when

$$
\mathbf{a}_{0}-2 \mathbf{a}_{1}+\mathbf{a}_{2}=\mathbf{b}_{0}-2 \mathbf{b}_{1}+\mathbf{b}_{2}=0, \quad \mathbf{a}_{N+1}-2 \mathbf{a}_{N}+\mathbf{a}_{N-1}=\mathbf{b}_{N+1}-2 \mathbf{b}_{N}+\mathbf{b}_{N-1}=0 .
$$

These additional conditions provide a system of $2 N+4$ equations in the $2 N+4$ complex variables $\mathbf{a}_{0}, \ldots, \mathbf{a}_{N+1}$ and $\mathbf{b}_{0}, \ldots, \mathbf{b}_{N+1}$. It should be noted that these equations are not algebraic, because the variables and their conjugates appear in them. They can be recast as a system of $4 N+8$ real equations in $4 N+8$ real variables (the real and imaginary parts of $\mathbf{a}_{0}, \ldots, \mathbf{a}_{N+1}$ and $\mathbf{b}_{0}, \ldots, \mathbf{b}_{N+1}$ ), but there is no guarantee of real solutions, because the equations are non-linear. 
In fact, preliminary experiments show that in most cases there are no solutions for which end segments with prescribed lengths are degree-elevated cubics (primarily due to the length constraint). Instead of end conditions, an approach based on shape optimization is used here to fix the residual degrees of freedom, similar to that employed in [10]. The idea is to rely on the good shape properties and simpler nature of $\mathrm{PH}$ cubic curves. As in [10], minimization of the global energy function

$$
F\left(\mathbf{a}_{0}, \mathbf{b}_{0}, \ldots, \mathbf{a}_{N+1}, \mathbf{b}_{N+1}\right):=\sum_{i=1}^{N}\left|\mathbf{a}_{i-1}-2 \mathbf{a}_{i}+\mathbf{a}_{i+1}\right|^{2}+\left|\mathbf{b}_{i-1}-2 \mathbf{b}_{i}+\mathbf{b}_{i+1}\right|^{2}
$$

will be considered. This can be regarded as a measure of the "distance" of the constructed $C^{2} \mathrm{PH}$ quintic spline from a $\mathrm{PH}$ cubic spline - in particular, minimization of (19) will exactly reproduce a PH cubic spline if the given points and segment arc lengths are compatible with such a curve. The problem is then one of optimization of the quadratic function (19), subject to the quadratic constraints defined by (15) and (17)-(18) for $i=1, \ldots, N$. In order to ensure rapid convergence, it is critical to have a good initial estimate for $\mathbf{a}_{0}, \ldots, \mathbf{a}_{N+1}$ and $\mathbf{b}_{0}, \ldots, \mathbf{b}_{N+1}$.

\subsection{Initialization of coefficients}

To obtain initial values for the coefficients $\mathbf{a}_{i}, \mathbf{b}_{i}$ the equations (15) and (17)-(18) are first simplified by setting $\Delta \mathbf{a}_{i}=\mathbf{a}_{i}-\mathbf{a}_{i-1}, \Delta \mathbf{b}_{i}=\mathbf{b}_{i}-\mathbf{b}_{i-1}$ for $i=1, \ldots, N$, substituting

$$
\begin{array}{ll}
\mathbf{a}_{i-1}=\mathbf{a}_{i}-\Delta \mathbf{a}_{i}, & \mathbf{a}_{i+1}=\mathbf{a}_{i}+\Delta \mathbf{a}_{i+1}, \\
\mathbf{b}_{i-1}=\mathbf{b}_{i}-\Delta \mathbf{b}_{i}, & \mathbf{b}_{i+1}=\mathbf{b}_{i}+\Delta \mathbf{b}_{i+1},
\end{array}
$$

into those equations, and retaining only zero-order terms in $\Delta \mathbf{a}_{i}, \Delta \mathbf{b}_{i}$. The equations (15) and (17)-(18) then yield

$$
\mathbf{a}_{i} \overline{\mathbf{b}}_{i}=\frac{1}{2}\left(\Delta y_{i}+\mathrm{i} \Delta z_{i}\right), \quad\left|\mathbf{a}_{i}\right|^{2}=\frac{1}{2}\left(l_{i}+\Delta x_{i}\right), \quad\left|\mathbf{b}_{i}\right|^{2}=\frac{1}{2}\left(l_{i}-\Delta x_{i}\right) .
$$

In order to ensure satisfaction of the compatibility condition

$$
\left|\mathbf{a}_{i} \overline{\mathbf{b}}_{i}\right|^{2}=\left|\mathbf{a}_{i}\right|^{2}\left|\mathbf{b}_{i}\right|^{2}
$$

equations (20) are then modified to

$$
\mathbf{a}_{i} \overline{\mathbf{b}}_{i}=\frac{1}{2} \gamma_{i}\left(\Delta y_{i}+\mathrm{i} \Delta z_{i}\right), \quad\left|\mathbf{a}_{i}\right|^{2}=\frac{1}{2} \gamma_{i}\left(\Delta p_{i}+\Delta x_{i}\right), \quad\left|\mathbf{b}_{i}\right|^{2}=\frac{1}{2} \gamma_{i}\left(\Delta p_{i}-\Delta x_{i}\right),
$$

where $\Delta p_{i}:=\left|\Delta \mathbf{p}_{i}\right|$ and $\gamma_{i}:=l_{i} / \Delta p_{i} \geq 1$. The solutions of equations (21) have the form

$$
\mathbf{a}_{i}=\sqrt{\frac{1}{2} \gamma_{i}\left(\Delta p_{i}+\Delta x_{i}\right)} \exp \left(\mathrm{i}\left(\theta_{i}+\phi_{i}\right)\right), \quad \mathbf{b}_{i}=\sqrt{\frac{1}{2} \gamma_{i}\left(\Delta p_{i}-\Delta x_{i}\right)} \exp \left(\mathrm{i} \phi_{i}\right),
$$

where $\phi_{i}$ is a free parameter, and $\theta_{i}$ is defined by

$$
\left(\cos \theta_{i}, \sin \theta_{i}\right):=\frac{\left(\Delta y_{i}, \Delta z_{i}\right)}{\sqrt{\left(\Delta y_{i}\right)^{2}+\left(\Delta z_{i}\right)^{2}}} .
$$

To determine the free parameters $\phi_{1}, \ldots, \phi_{N}$ we first set $\phi_{1}=0$, and then obtain $\phi_{i}$ from $\phi_{i-1}$ for $i=2, \ldots, N$ by minimizing $\left|\Delta \mathbf{a}_{i}\right|^{2}+\left|\Delta \mathbf{b}_{i}\right|^{2}$ with respect to it. We have

$$
\begin{aligned}
\left|\Delta \mathbf{a}_{i}\right|^{2}+\left|\Delta \mathbf{b}_{i}\right|^{2}= & \gamma_{i-1} \Delta p_{i-1}+\gamma_{i} \Delta p_{i} \\
& -\sqrt{\gamma_{i-1} \gamma_{i}\left(\Delta p_{i-1}-\Delta x_{i-1}\right)\left(\Delta p_{i}-\Delta x_{i}\right)} \cos \left(\Delta \phi_{i}\right) \\
& -\sqrt{\gamma_{i-1} \gamma_{i}\left(\Delta p_{i-1}+\Delta x_{i-1}\right)\left(\Delta p_{i}+\Delta x_{i}\right)} \cos \left(\Delta \theta_{i}+\Delta \phi_{i}\right),
\end{aligned}
$$


where $\Delta \theta_{i}=\theta_{i}-\theta_{i-1}$ and $\Delta \phi_{i}=\phi_{i}-\phi_{i-1}$. Setting the derivative of this expression with respect to $\Delta \phi_{i}$ equal to zero yields

$$
\tan \Delta \phi_{i}=\frac{-\sin \Delta \theta_{i}}{\cos \Delta \theta_{i}+\sqrt{\frac{\left(\Delta p_{i-1}-\Delta x_{i-1}\right)\left(\Delta p_{i}-\Delta x_{i}\right)}{\left(\Delta p_{i-1}+\Delta x_{i-1}\right)\left(\Delta p_{i}+\Delta x_{i}\right)}}} .
$$

This identifies two values $\Delta \phi_{i} \in(-\pi,+\pi]$ corresponding to minimum and maximum values of $\left|\Delta \mathbf{a}_{i}\right|^{2}+\left|\Delta \mathbf{b}_{i}\right|^{2}$, and we must distinguish the former from the latter by evaluating this expression. Obtaining $\phi_{i}$ from $\Delta \phi_{i}$, the values $\mathbf{a}_{i}$ and $\mathbf{b}_{i}$ are then completely determined from (22). Figure 2 compares the curves generated by the coefficients $\mathbf{a}_{0}, \ldots, \mathbf{a}_{N+1}$ and $\mathbf{b}_{0}, \ldots, \mathbf{b}_{N+1}$ obtained from the above initialization scheme, with that generated by a random choice of the coefficients. The former is clearly much closer to interpolating the prescribed data points and segment arc lengths, resulting in much faster convergence to the desired interpolant - see Section 4.6 below.
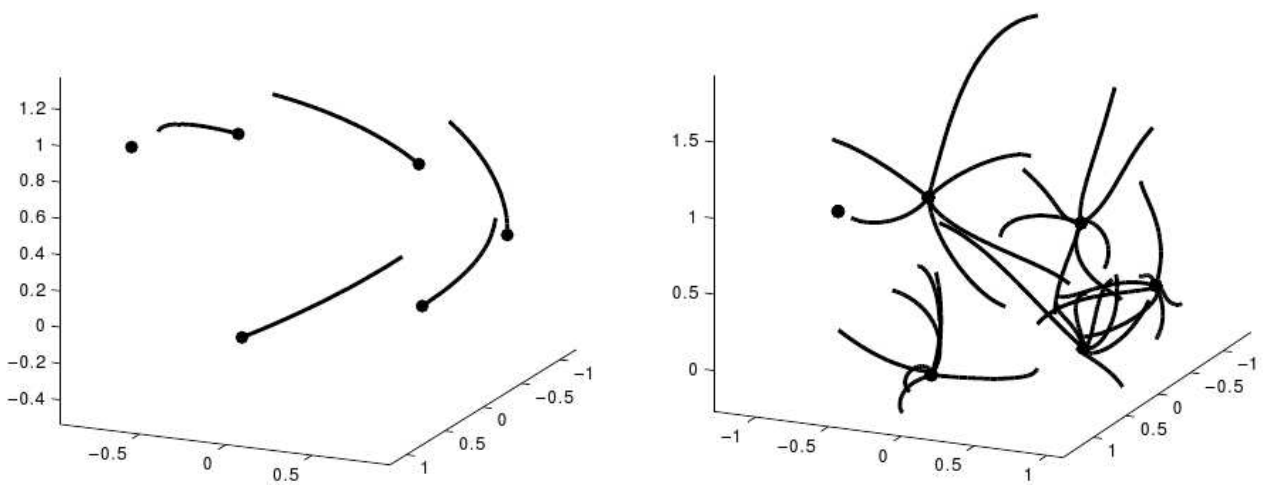

Figure 2: PH quintic spline curves defined by coefficients $\mathbf{a}_{0}, \ldots, \mathbf{a}_{N+1}$ and $\mathbf{b}_{0}, \ldots, \mathbf{b}_{N+1}$ generated by the initialization scheme of Section 4.4 (left) and by a set of randomly-chosen coefficients (right). The six data points correspond to parameter values $t=0.0,0.5,1.0,1.5,2.0,2.5$ on the circular helix $\mathbf{r}(t)=(\cos 2 t, \sin 2 t, 0.3 t)$, with exact helix segment arc lengths used for the initialization scheme.

\subsection{Computational procedure}

The examples presented below were all computed using the interior-point algorithm of the MATLAB "fmincon" constrained-optimization function. The objective function (19) is quadratic and convex in the variables $\mathbf{a}_{0}, \mathbf{b}_{0}, \ldots, \mathbf{a}_{N+1}, \mathbf{b}_{N+1}$. The constraints (15) and (17)-(18) are also quadratic, but non-convex. The efficiency of the method is enhanced by exploiting the fact that the Jacobian and Hessian matrices for the cost function and the constraints are sparse and have simple, closed-form expressions. All of the calculations were performed on a $\mathrm{PC}$ with a $3 \mathrm{GHz}$ cpu.

It should be noted that solutions may not exist for arbitrarily-chosen data. In particular, when the points $\mathbf{p}_{0}, \ldots, \mathbf{p}_{N}$ are sampled from a smooth analytic curve, the experiments indicate that 
the specified segment arc lengths $l_{1}, \ldots, l_{N}$ should be "close" to those of the analytic curve, to ensure existence of a solution. Because of the non-linear nature of the problem, it is difficult to formulate precise a priori "closeness" criteria to guarantee the existence of a solution. The segment arc lengths should therefore be regarded as parameters that can be interactively adjusted by the user to ensure the existence of an interpolant with desirable shape properties.

For admissible points and arc-length constraints, the optimization process is found to be robust and rapidly convergent to machine precision, so the prescribed points and arc lengths between them are precisely matched. Although convergence is often observed without invoking the initialization step described in Section 4.4, this step is strongly preferred because (i) convergence is much faster; and (ii) the likelihood of converging to a non-optimal local minimum is greatly reduced.

\subsection{Numerical examples}

Two kinds of applications are considered here, with corresponding initial data sets.

(a) In the first application context, the data (points $\mathbf{p}_{i}$ and segment lengths $l_{i}$ ) come from physical measurements, and correspond to a real curve. The data are therefore physically and geometrically coherent, and the interpolation scheme corresponds to a reconstruction problem that aims to find a solution close to the physical curve. Such initial data may, for instance, be obtained by a physical shape sensing system similar to the Morphosense ribbon. In this context, two key observations arise from an analysis of the computed examples.

- The $C^{2} \mathrm{PH}$ quintic spline interpolation under segment length constraints using exact data allows more faithful curve reconstruction than classical interpolation methods. As is evident in Figure 3, the method is a good alternative to a costly refinement of the sampling: when prescribing lengths close to the actual lengths of the sampled curve, the method provides a $C^{2}$ spline curve closer to the original curve than the natural $C^{2}$ cubic spline.

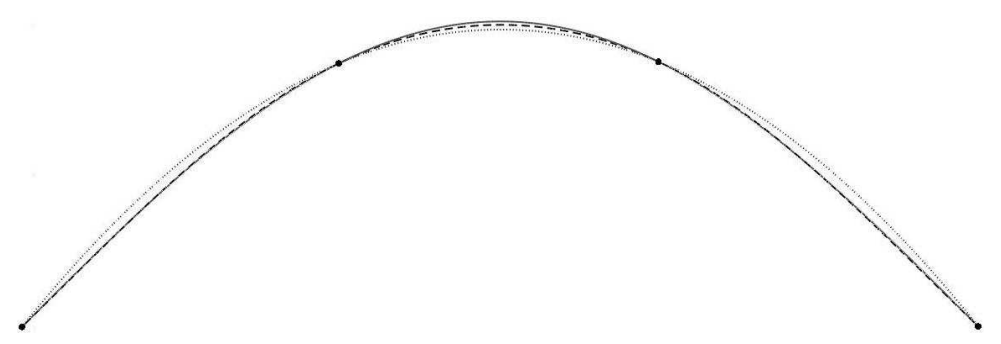

Figure 3: Approximation of a sine curve (solid) by a $C^{2}$ cubic spline (dotted) interpolating discrete points, and by a $C^{2} \mathrm{PH}$ quintic spline (dashed) interpolating both points and segment arc lengths. The data points correspond to the parameter values $t=0, \frac{1}{3} \pi, \frac{2}{3} \pi, \pi$ on the curve $\mathbf{r}(t)=(t, \sin t, 0)$.

- If the data are intrinsically constrained by a strong underlying structure (the Morphosense, for instance, follows geodesic curves of the surface on which it is placed), the method gives good results when using the actual lengths between the knots. However, when the lengths are modified even slightly, the reconstructed curves may exhibit undesired oscillations that are often difficult to 
control. Figure 4 illustrates this in the case of the data points $t=0.0,0.5,1.0,1.5,2.0,2.5$ uniformly sampled from the circular helix $\mathbf{r}(t)=(\cos 2 t, \sin 2 t, 0.3 t)$, using both the exact helix arc lengths, and arc lengths deviating by $\pm 1 \%$ from the exact values.

(a)

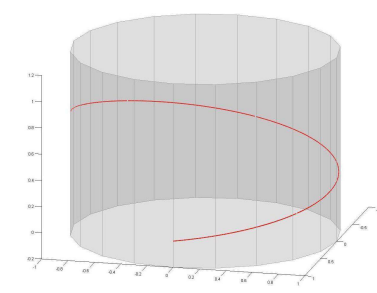

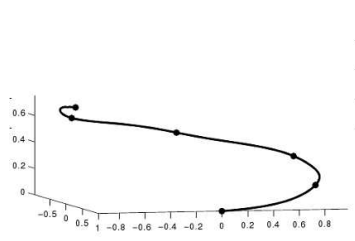

(b)
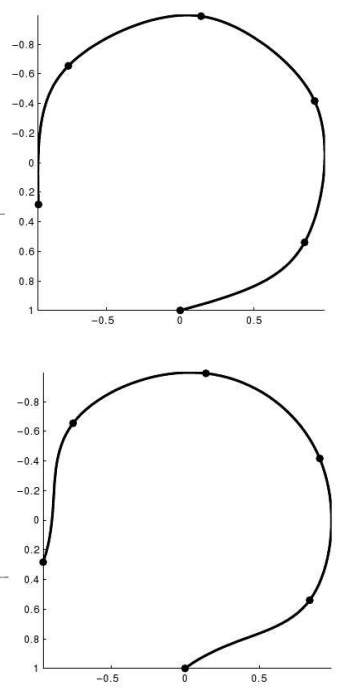

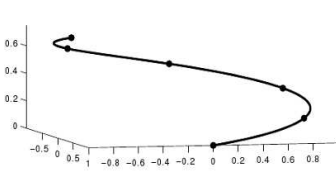

(c)
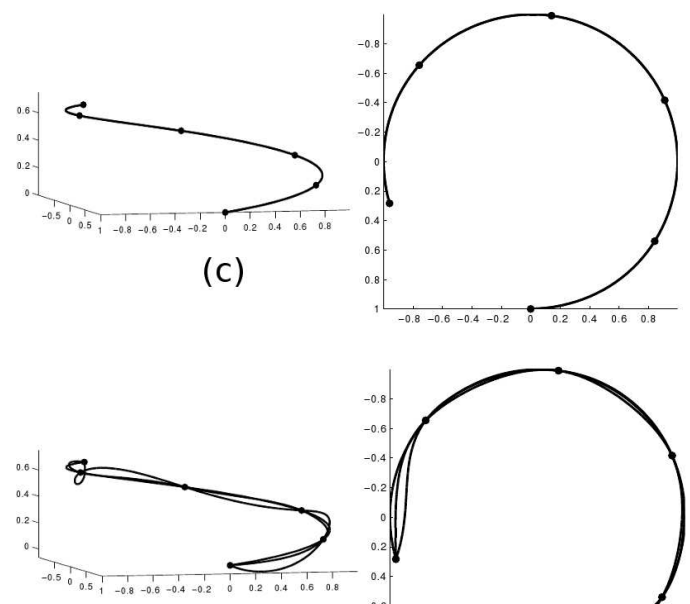

(e)

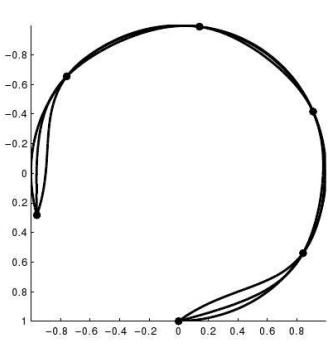

Figure 4: (a) A helix with known segment lengths $L_{i}$ between sample points $\mathbf{p}_{0}, \ldots, \mathbf{p}_{5}$. Also shown are reconstructions interpolating the given points with segment lengths (b) $l_{i}=0.99 L_{i}$; (c) $l_{i}=L_{i}$; and (d) $l_{i}=1.01 L_{i}$ illustrated as $(x, z)$ and $(x, y)$ plots. (e) Superposition of the reconstructions.

Table 1 lists, for this example, the number of iterations and cpu time required for convergence of the optimization process to machine precision, with and without use of the initialization scheme described in Section 4.4. Although the optimization typically converges with even "random" choices for the initial values, it is apparent that the method of Section 4.4 can substantially accelerate the convergence (and is more likely to identify the global minimum). Table 2 illustrates how the number of iterations and cpu time required for convergence to machine precision vary with the number of data points uniformly sampled from the circular helix, using the initialization scheme of Section 4.4.

(b) In a different context, one may wish to only interpolate the points $\mathbf{p}_{i}$ and exploit the segment length constraints $l_{i}$ as "tension" or "design" parameters. In this case, the lengths $l_{i}$ must be chosen carefully. Obviously, the lengths must satisfy the lower bounds $l_{i} \geq\left|\Delta \mathbf{p}_{i}\right|$, and excessive lengths can introduce undesired behavior (such as loops). Figure 5 shows a case in which different lengths are chosen for the second segment, resulting in two radically different solutions for the curve (even 


\begin{tabular}{|c|cc|cc|}
\hline & \multicolumn{2}{|c|}{ with initialization } & \multicolumn{2}{c|}{ without initialization } \\
& iterations & cpu time & iterations & cpu time \\
\hline$l_{i}=0.99 L_{i}$ & 30 & 0.24 sec. & 130 & $0.85 \mathrm{sec}$. \\
$l_{i}=1.00 L_{i}$ & 49 & $0.29 \mathrm{sec}$. & 135 & $0.67 \mathrm{sec}$. \\
$l_{i}=1.01 L_{i}$ & 24 & $0.17 \mathrm{sec}$. & 124 & $0.73 \mathrm{sec}$. \\
\hline
\end{tabular}

Table 1: Number of iterations for convergence to machine precision and compute times required for the helix example in Figure 4, with and without the initialization procedure described in Section 4.4.

\begin{tabular}{|c|cc|}
\hline data points & iterations & cpu time \\
\hline 4 & 15 & $0.12 \mathrm{sec}$. \\
6 & 37 & $0.33 \mathrm{sec}$. \\
8 & 45 & $0.47 \mathrm{sec}$. \\
10 & 62 & $0.80 \mathrm{sec}$ \\
15 & 69 & $1.03 \mathrm{sec}$. \\
20 & 141 & $3.14 \mathrm{sec}$. \\
\hline
\end{tabular}

Table 2: Dependence of number of required iterations and total cpu times on the number of sampled data points for the circular helix example, using the initialization scheme described in Section 4.4.
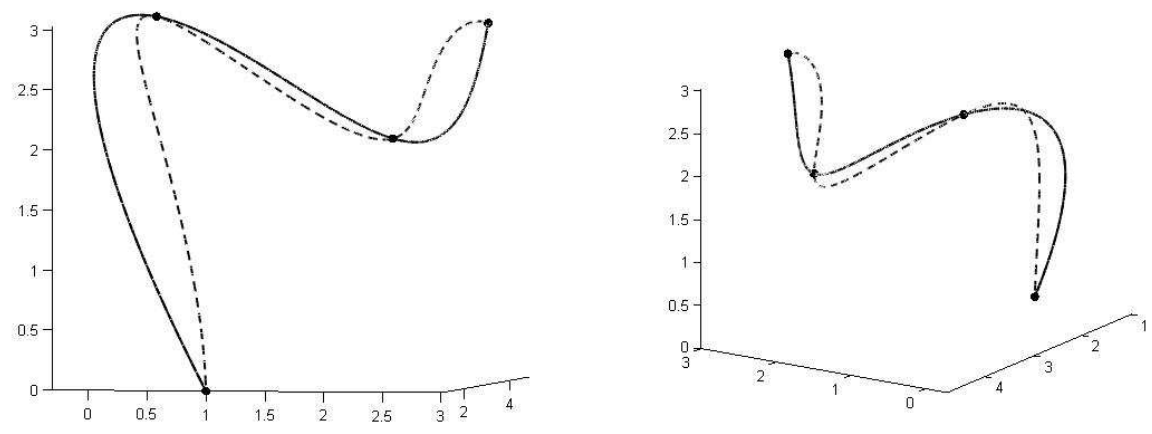

Figure 5: Two views of an example interpolating points $\mathbf{p}_{0}=(1,1,0), \mathbf{p}_{1}=(0,4,3), \mathbf{p}_{2}=(2,4,2)$, $\mathbf{p}_{3}=(3,3,3)$ with equal lengths $l_{1}=4.9$ and $l_{3}=2.0$ for the first and last segment but the different values $l_{2}=2.9$ (dashed curve) and $l_{2}=2.5$ (solid curve) for the middle segment (a $16 \%$ difference).

for adjacent segments of fixed length). Figure 6 shows another case, in which three different PH quintic splines interpolating the points

$$
\mathbf{p}_{0}=(1,1,0), \quad \mathbf{p}_{1}=(0,4,3), \quad \mathbf{p}_{2}=(2,1,1), \quad \mathbf{p}_{3}=(2,4,2), \quad \mathbf{p}_{4}=(3,3,3)
$$


are constructed, with fixed segment lengths $l_{2}=5.0, l_{3}=3.9, l_{4}=2.75$, and three different values for $l_{1}$. This example illustrates the use of the segment length as a design parameter. The number of iterations and total cpu time required for convergence to machine precision are listed in Table 3 , with and without the initialization procedure described in Section 4.4.
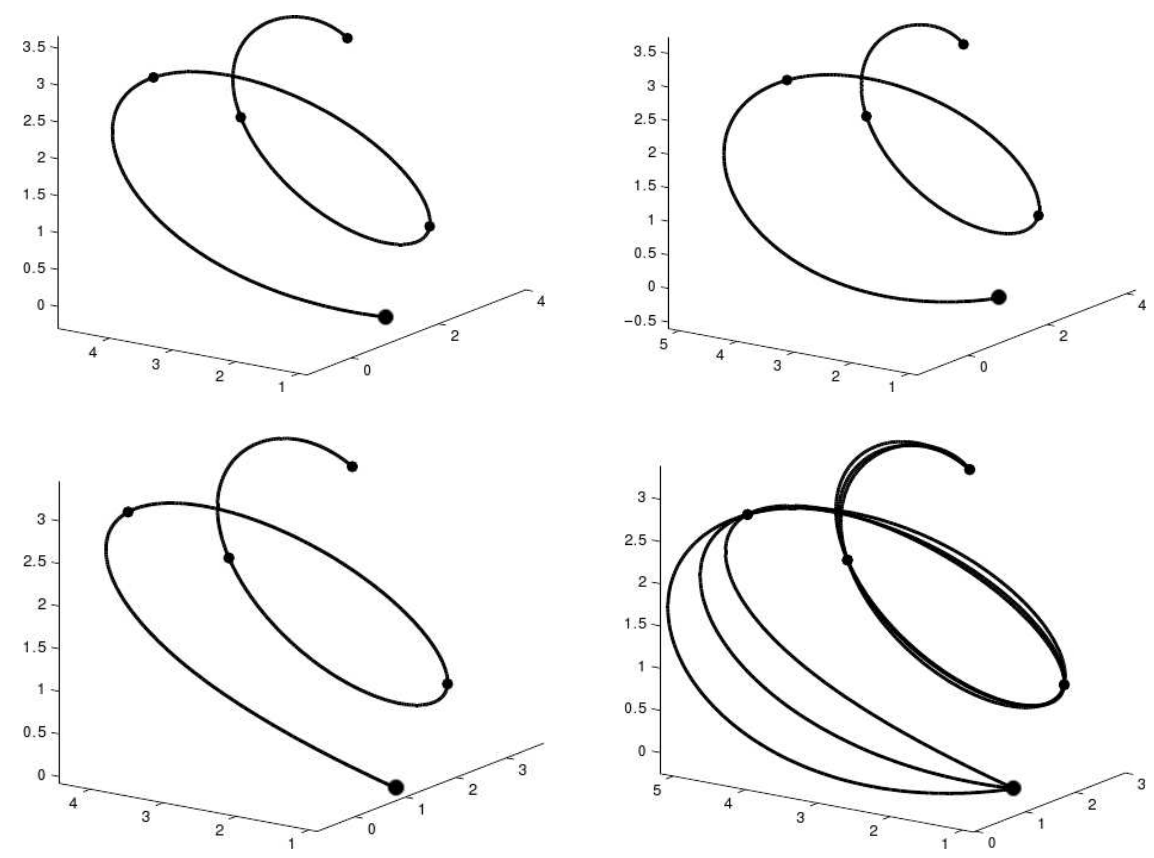

Figure 6: Examples of interpolants to fixed points and equal segment lengths $l_{2}, l_{3}, l_{4}$ but different $l_{1}$ values. Upper left curve: $l_{1}=6.3$. Upper right curve: $l_{1}$ is increased by $15 \%$ to 7.24 . Lower left curve: $l_{1}$ is decreased by $15 \%$ to 5.35 . Lower right: all three of the interpolants are superimposed.

\begin{tabular}{|c|c|c|c|c|}
\hline & \multicolumn{2}{|c|}{$\begin{array}{l}\text { with initialization } \\
\text { iterations cpu time }\end{array}$} & \multicolumn{2}{|c|}{$\begin{array}{l}\text { without initialization } \\
\text { iterations cpu time }\end{array}$} \\
\hline case (a) & 31 & $0.16 \mathrm{sec}$ & 90 & 0.48 sec. \\
\hline case (b) & 27 & $0.14 \mathrm{sec}$. & 99 & $0.51 \mathrm{sec}$ \\
\hline case (c) & 25 & $0.12 \mathrm{sec}$. & 91 & $0.50 \mathrm{sec}$ \\
\hline
\end{tabular}

Table 3: Number of iterations for convergence to machine precision and compute times required for the example in Figure 6, with and without use of the initialization scheme described in Section 4.4.

It should be noted that solutions may not exist when the prescribed segment arc lengths $l_{i}$ are large compared to the spacings $\Delta p_{i}$ between the points - and, if they do exist, they are generally of undesirable shape. Figure 7 illustrates this in the case of the four equidistant points

$$
\mathbf{p}_{0}=(0,0,0), \quad \mathbf{p}_{1}=(0,1,0), \quad \mathbf{p}_{2}=(1,1,0), \quad \mathbf{p}_{3}=(1,1,1),
$$


with one or more of the ratios $\gamma_{i}=l_{i} / \Delta p_{i}$ being large compared to unity. For comparison, the case $\left(\gamma_{1}, \gamma_{2}, \gamma_{3}\right)=(1.15,1.08,1.15)$ is also shown in Figure 7 - this corresponds to the solution of the optimization problem when the length constraints are relaxed.


Figure 7: Examples of $C^{2} \mathrm{PH}$ quintic splines interpolating the points (23) with prescribed segment lengths $l_{i}$ that substantially exceed the spacings $\Delta p_{i}$ between successive points (dashed curves). The ratios $\gamma_{i}=l_{i} / \Delta p_{i}$ have the values $\left(\gamma_{1}, \gamma_{2}, \gamma_{3}\right)=(1.1,2,10)$ on the left; $(5,2,5)$ in the center; and $(10,3,10)$ on the right. The solid "reference" curve corresponds to $\left(\gamma_{1}, \gamma_{2}, \gamma_{3}\right)=(1.15,1.08,1.15)$.

Finally, the existence of solutions that correspond to local minima of the objective function is illustrated in Figure 8. The four data points for this example are the same as in Figure 5, and the prescribed segment lengths are $l_{1}=9.25, l_{2}=4.75, l_{3}=3.70$. When the initialization scheme of Section 4.4 is employed, the optimization converges to the global minimum, but may otherwise converge to one of the solutions representing a local minimum.

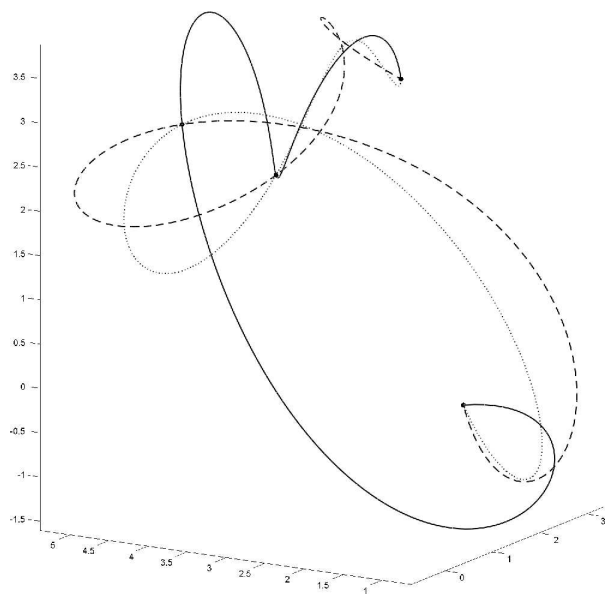

Figure 8: Interpolants corresponding to the global minimum (solid curve) and local minima (dashed and dotted curves) of the objective function. The data points are as in Figure 5 with segment lengths $\left(l_{1}, l_{2}, l_{3}\right)=(9.25,4.75,3.70)$. The initialization scheme ensures convergence to the global minimum. 


\section{$5 \quad C^{2}$ interpolation of derivatives under length constraints}

We consider now the interpolation of derivative vectors $\mathbf{d}_{0}, \ldots, \mathbf{d}_{N}$ (rather than points $\left.\mathbf{p}_{0}, \ldots, \mathbf{p}_{N}\right)$ by $C^{2} \mathrm{PH}$ quintic splines, subject to prescribed lengths $l_{1}, \ldots, l_{N}$ for each segment.

\subsection{Interpolation of derivatives}

Given derivative vectors $\mathbf{d}_{0}, \ldots, \mathbf{d}_{N}$ and prescribed segment lengths $l_{1}, \ldots, l_{N}$ we wish to compute a $C^{2} \mathrm{PH}$ quintic spline, as defined in Section 3, with segments $\mathbf{r}_{i}(t)$ for $i=1, \ldots, N$ such that

$$
\begin{aligned}
& \text { (a) } \mathbf{r}_{i}(1)=\mathbf{r}_{i+1}(0) \quad \text { for } i=1, \ldots, N-1, \\
& \text { (b) } \mathbf{r}_{1}^{\prime}(0)=\mathbf{d}_{0}, \quad \mathbf{r}_{N}^{\prime}(1)=\mathbf{d}_{N}, \quad \mathbf{r}_{i}^{\prime}(1)=\mathbf{r}_{i+1}^{\prime}(0)=\mathbf{d}_{i} \quad \text { for } i=1, \ldots, N-1, \\
& \text { (c) } \int_{0}^{1}\left|\mathbf{r}_{i}^{\prime}(t)\right| \mathrm{d} t=l_{i} \quad \text { for } i=1, \ldots, N .
\end{aligned}
$$

As with the previous method, we note that condition (a), together with the constructions introduced in Section 3, automatically generates a $C^{2} \mathrm{PH}$ quintic spline. Each of the conditions (a) $-(\mathrm{c})$ is considered in greater detail below.

(a) This condition simply states that the spline must have positional continuity, i.e., each segment $\mathbf{r}_{i+1}(t)$ must begin where the previous segment $\mathbf{r}_{i}(t)$ ends. This is achieved by taking $\mathbf{r}_{i+1}(0)=\mathbf{r}_{i}(1)$ as the integration constant upon integrating $\mathbf{r}_{i+1}^{\prime}(t)$. Thus, only the first integration constant $\mathbf{r}_{1}(0)$ is free, corresponding to a translational freedom of the solution.

(b) Since PH curves are generated by integrating their hodograph, this condition can be formulated directly from equation (9) as

$$
\frac{1}{4}\left(\left|\mathbf{a}_{i}+\mathbf{a}_{i+1}\right|^{2}-\left|\mathbf{b}_{i}+\mathbf{b}_{i+1}\right|^{2}\right)=d x_{i}, \quad \frac{1}{2}\left(\mathbf{a}_{i}+\mathbf{a}_{i+1}\right)\left(\overline{\mathbf{b}}_{i}+\overline{\mathbf{b}}_{i+1}\right)=d y_{i}+\mathrm{i} d z_{i},
$$

where $\mathbf{d}_{i}=\left(d x_{i}, d y_{i}, d z_{i}\right)$ for $i=0, \ldots, N$. As with the previous method, the first of equations (24) is real, while the second is complex. The relations (24) thus amount to $3(N+1)$ scalar equations (three for each interpolated derivative vector).

(c) As in the previous method, the $N$ equations (16) expressing the length constraints are imposed.

The system defined by (16) and (24) comprises $4 N+3$ scalar equations, to be satisfied by the $4 N+8$ scalar freedoms embodied in the complex coefficients $\mathbf{a}_{0}, \ldots, \mathbf{a}_{N+1}$ and $\mathbf{b}_{0}, \ldots, \mathbf{b}_{N+1}$. This problem is therefore somewhat more constrained than that of Section 4 - three additional constraints arise from the interpolation of the initial derivative $\mathbf{d}_{0}$. In the previous method, interpolation of the first point was achieved by choice of the integration constant, but this is not possible for interpolation of the initial derivative vector in the present context. As in Section 4, the spline computation will be based on minimization of the function (19) under the constraints (16) and (24).

\subsection{Initialization of coefficients}

The initialization method is analogous to that employed in Section 4. Substituting $\mathbf{a}_{i-1}=\mathbf{a}_{i}-\Delta \mathbf{a}_{i}$, $\mathbf{a}_{i+1}=\mathbf{a}_{i}+\Delta \mathbf{a}_{i+1}$, and $\mathbf{b}_{i-1}=\mathbf{b}_{i}-\Delta \mathbf{b}_{i}, \mathbf{b}_{i+1}=\mathbf{b}_{i}+\Delta \mathbf{b}_{i+1}$ in equations (16) and (24), and retaining only the zero order terms, we obtain the simplified equation system

$$
\left|\mathbf{a}_{i}\right|^{2}+\left|\mathbf{b}_{i}\right|^{2}=l_{i}, \quad\left|\mathbf{a}_{i}\right|^{2}-\left|\mathbf{b}_{i}\right|^{2}=d x_{i}, \quad \mathbf{a}_{i} \overline{\mathbf{b}}_{i}=\frac{1}{2}\left(d y_{i}+\mathrm{i} d z_{i}\right) .
$$


By combining the first and second equations, these can be re-formulated as

$$
\left|\mathbf{a}_{i}\right|^{2}=\frac{1}{2}\left(l_{i}+d x_{i}\right), \quad\left|\mathbf{b}_{i}\right|^{2}=\frac{1}{2}\left(l_{i}-d x_{i}\right), \quad \mathbf{a}_{i} \overline{\mathbf{b}}_{i}=\frac{1}{2}\left(d y_{i}+\mathrm{i} d z_{i}\right) .
$$

This system is similar to the simplified equations (20) for the previous method, and can be viewed as a "continuous" version of the latter, with the the derivatives $\left(d x_{i}, d y_{i}, d z_{i}\right)$ replacing the differences $\left(\Delta x_{i}, \Delta y_{i}, \Delta z_{i}\right)$. Figure 9 shows an example curve generated by the initial approximation.

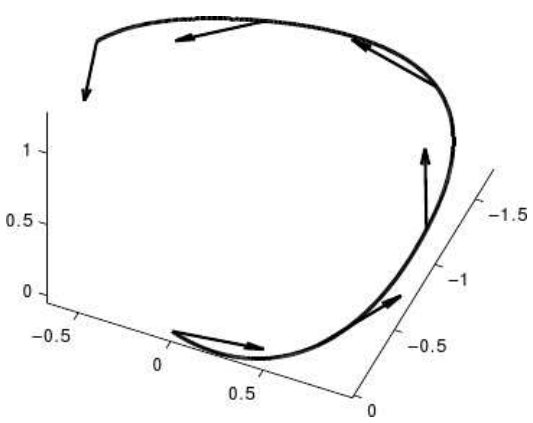

Figure 9: Curve determined by the initial values for derivative and arc length data from a helix.

\subsection{Numerical examples}

The computational procedure employed in the present context is, with appropriate modifications, as described in Section 4.5. We consider again both curve reconstruction and curve design contexts. The derivative interpolation scheme described above was motivated by the Morphosense shape reconstruction problem. However, the Morphosense provides unit orientation vectors, rather than derivatives of non-unit magnitude. Several methods are currently being investigated to address this issue. Figure 10 shows preliminary results obtained from noisy data measured by a Morphosense. For this example, the normalized derivatives obtained from the sensors are

$$
\begin{array}{lll}
\mathbf{d}_{0}=(0.3862,-0.0315,0.9219), & \mathbf{d}_{1}=(0.3775,-0.1613,0.9118), \\
\mathbf{d}_{2}=(0.4077,-0.1947,0.8921), & \mathbf{d}_{3}=(0.4444,-0.3071,0.8415), \\
\mathbf{d}_{4}=(0.3224,-0.4485,0.8336), & \mathbf{d}_{5}=(0.3913,-0.4889,0.7797), \\
\mathbf{d}_{6}=(0.2771,-0.5945,0.7548), & \mathbf{d}_{7}=(0.1710,-0.7053,0.6880),
\end{array}
$$

and the measured distances between sensors are $l_{1}=170.05, l_{2}=169.50, l_{3}=168.26, l_{4}=169.33$, $l_{5}=169.53, l_{6}=168.82, l_{7}=170.10$. For the reconstruction, the vectors $\mathbf{d}_{i}$ are scaled by $\frac{1}{2}\left(l_{i}+l_{i+1}\right)$ for $i=1, \ldots, 6$ while $\mathbf{d}_{0}$ and $\mathbf{d}_{7}$ are scaled by $l_{1}$ and $l_{7}$ respectively. With initialization, the solution converged in 31 iterations and 0.28 seconds ( 260 iterations and 2.25 seconds without initialization). These convergence figures, with initialization, are representative of all subsequent examples.

Figure 11 illustrates a reconstruction from arc lengths and unit tangent vectors sampled from the circular helix $\mathbf{r}(t)=(\cos 2 t, \sin 2 t, 0.3 t)$ at parameter values $t=0.0,0.5,1.0,1.5,2.0,2.5$ (compare with Figure 4 ). The unit tangents are scaled by the segment arc lengths as in the preceding example. 

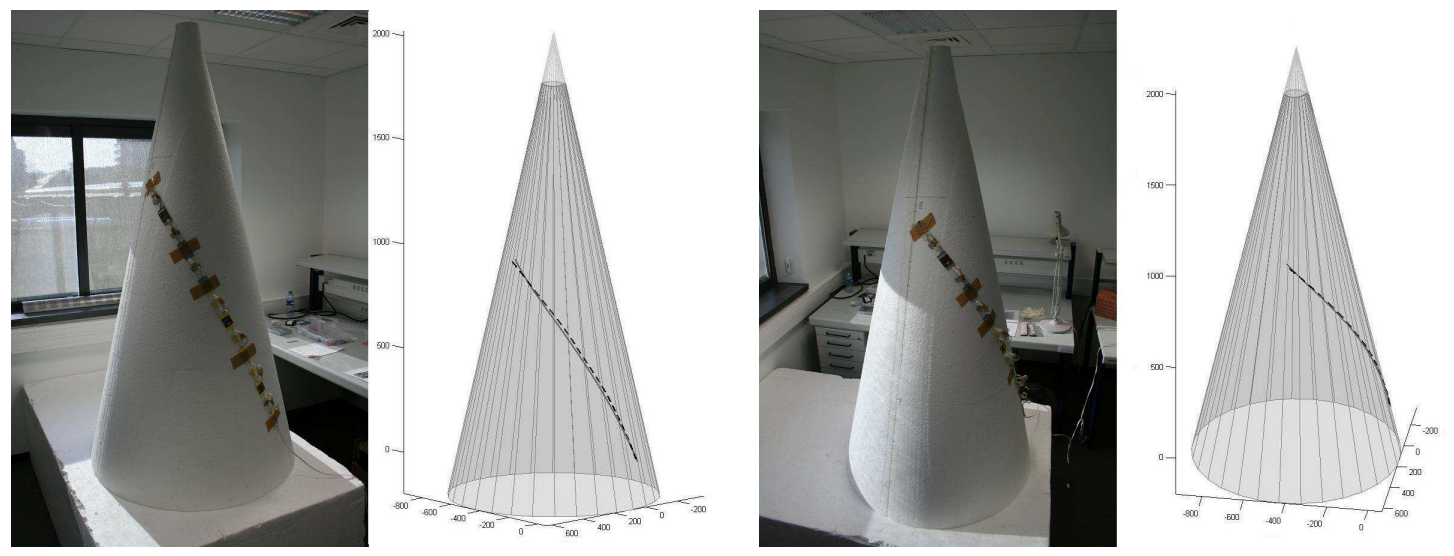

Figure 10: Acquisition and reconstruction of a curve on a cone with the Morphosense ribbon. The analytical curve (solid) is acquired by the ribbon and reconstructed as a PH quintic spline (dashed).

(a)
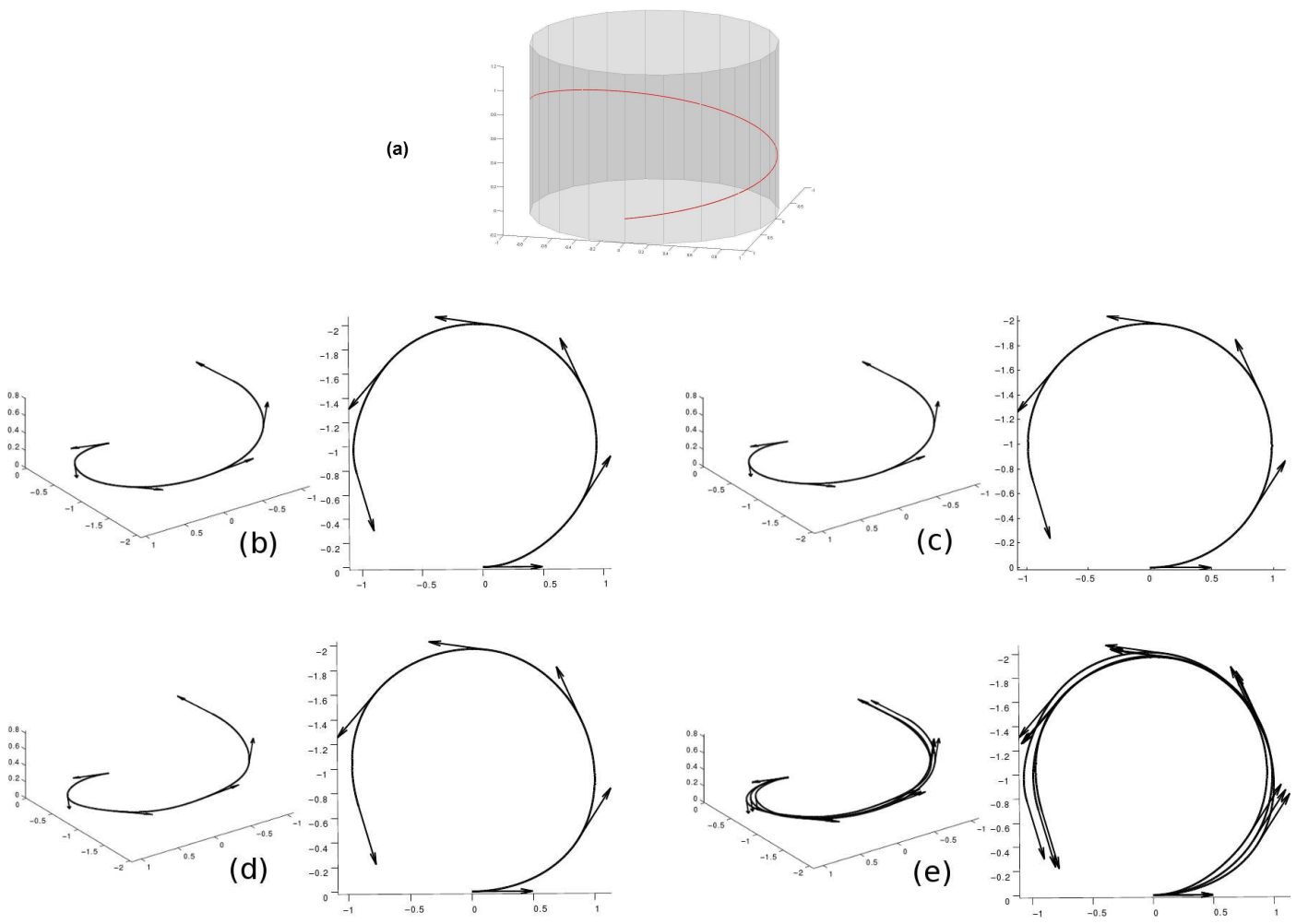

Figure 11: (a) A helix with known segment lengths $L_{i}$ between sampled derivatives $\mathbf{d}_{0}, \ldots, \mathbf{d}_{5}$. Reconstructions interpolating the given derivatives with segment lengths (b) $l_{i}=0.99 L_{i}$; (c) $l_{i}=L_{i}$; and (d) $l_{i}=1.01 L_{i}$ are illustrated as $(x, z)$ and $(x, y)$ plots. (e) Superposition of the reconstructions. 
As with the point interpolation method of Section 4, the shape is seen to be quite sensitive to the prescribed length constraints. If the specified segment lengths differ significantly from those of the analytical curve, the interpolant exhibits strong oscillations.
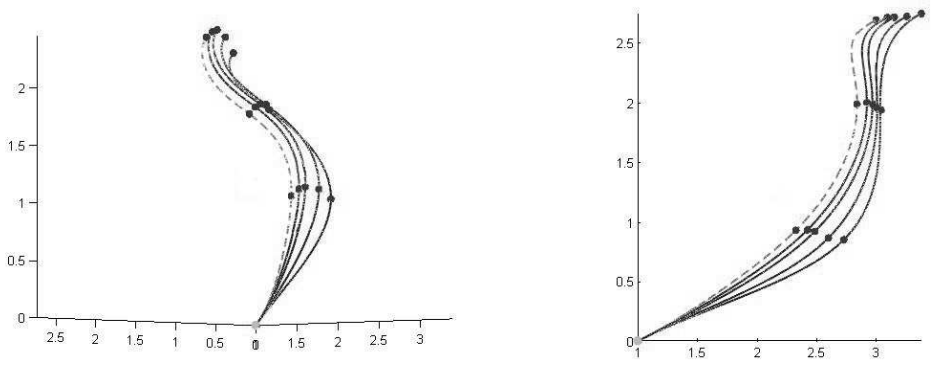

Figure 12: An example with different lengths $l_{1}$ for the first segment. For the dashed curve, the first segment has length $l_{1}=2$, and this is increased by $1 \%$ for each of the successive (solid) curves.

Figures 12 and 13 show examples of reconstructions from arbitrarily-chosen derivative vectors and arc lengths, illustrating again the strong influence of the length constraints on the curve shape. In Figure 12, the derivatives and segment arc lengths are $\mathbf{d}_{0}=(2,1,1), \mathbf{d}_{1}=(1,1,1), \mathbf{d}_{2}=(0,1,0)$, $\mathbf{d}_{3}=\left(1, \frac{1}{2}, \frac{1}{2}\right)$ and $l_{1}=2.0, l_{2}=1.4, l_{3}=1.0$, with $l_{1}$ being increased by $1 \%$ for successive curves. In the Figure 13 example, we choose $\mathbf{d}_{0}=(-2,-1,-1), \mathbf{d}_{1}=\left(1, \frac{1}{2}, \frac{1}{2}\right), \mathbf{d}_{2}=\left(0,1, \frac{1}{2}\right), \mathbf{d}_{3}=\left(0,-1,-\frac{1}{2}\right)$ and $l_{1}=1.2, l_{2}=1.4, l_{3}=0.7$, with $l_{2}$ being increased by $1 \%$ for successive curves. The segment lengths evidently serve as useful tension parameters, facilitating manipulation of the curve shape in geometric design applications.
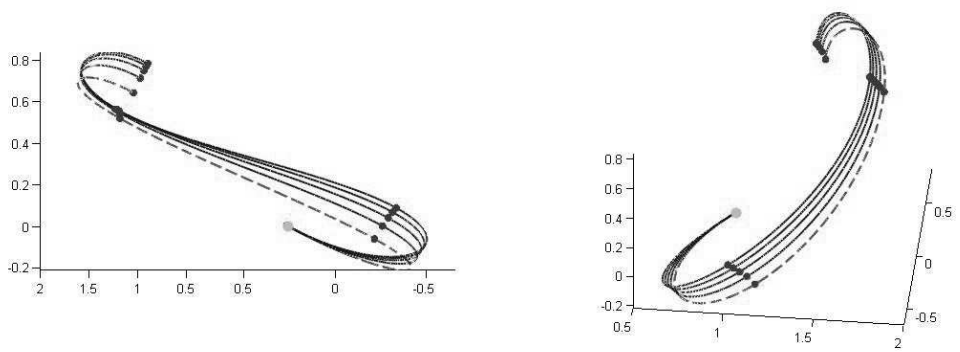

Figure 13: An example with different lengths $l_{2}$ for the second segment. For the dashed curve, the second segment has length $l_{2}=1.4$. This increases by $1 \%$ for each of the successive (solid) curves.

Finally, Figure 14 illustrates the behavior of interpolants to prescribed derivatives and segment arc lengths in the case of "large" lengths. As a reference case, the arc lengths are specified as 
$l_{1}=l_{2}=l_{3}=1$ and the derivatives are defined by the unit vectors

$$
\mathbf{d}_{0}=(1,0,0), \quad \mathbf{d}_{1}=(0,1,0), \quad \mathbf{d}_{2}=(0,0,1), \quad \mathbf{d}_{3}=(1,0,0) .
$$

When the segment lengths are increased to $l_{1}, l_{2}, l_{3}=3$ (without changing $\mathbf{d}_{0}, \mathbf{d}_{1}, \mathbf{d}_{2}, \mathbf{d}_{3}$ ) the overall size of the curve increases, but the individual segments become very "flat" because of the relatively small derivative magnitudes. However, if $\mathbf{d}_{0}, \mathbf{d}_{1}, \mathbf{d}_{2}, \mathbf{d}_{3}$ are also scaled by a factor of 3 , the resulting interpolant is simply a uniformly-scaled instance of the reference curve. This behavior, apparent in Figure 14, illustrates the importance of using commensurate derivative magnitudes and segment arc lengths to ensure interpolants of good shape quality.
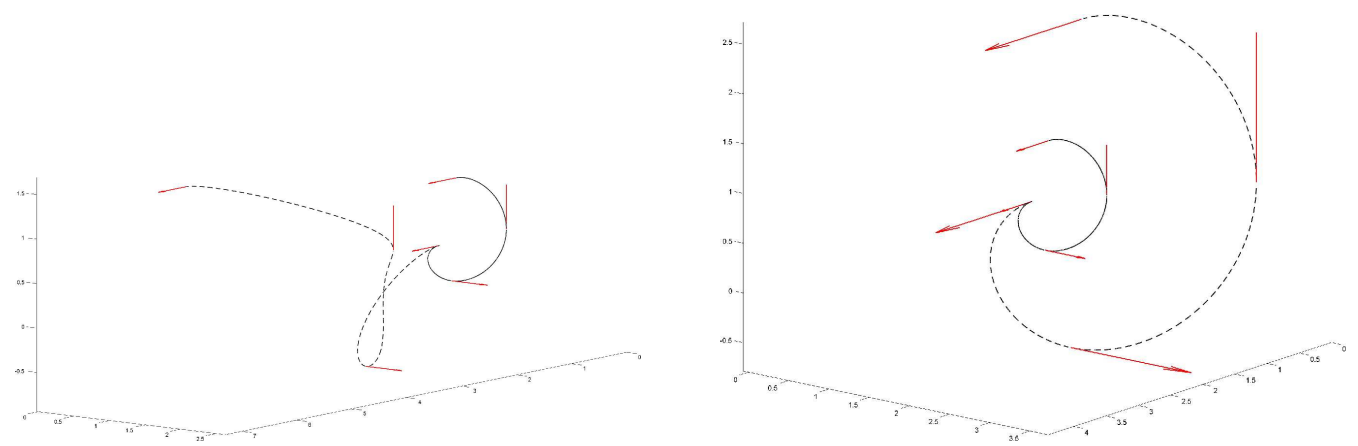

Figure 14: Left: the interpolants to the derivatives (27) with segment lengths $l_{1}=l_{2}=l_{3}=1$ and $l_{1}=l_{2}=l_{3}=3$. Right: interpolant to the derivatives (27) with segment lengths $l_{1}=l_{2}=l_{3}=1$ compared with the interpolant when $\mathbf{d}_{0}, \mathbf{d}_{1}, \mathbf{d}_{2}, \mathbf{d}_{3}$ and $l_{1}, l_{2}, l_{3}$ are both scaled by a factor of 3 .

\section{Conclusion}

Two novel $C^{2}$ curve interpolation schemes, motivated by applications to shape reconstruction from discrete micro-electronic sensor data, have been proposed. A distinctive feature of these methods is the satisfaction of prescribed segment length constraints, based on the special algebraic structure of spatial Pythagorean-hodograph $(\mathrm{PH})$ curves. This feature is very important in contexts where the precise spacing between sensors is known, and also provides useful shape or tension parameters for manipulating curves in geometric design contexts. It is expected that many other physical or geometrical modelling applications can also benefit from this capability.

The interpolation schemes involve the optimization of a quadratic cost function, subject to a system of quadratic constraints, and methods to identify a good starting approximation ensure an efficient and robust iterative solution of this problem. Computed examples show that the prescribed segment lengths strongly influence the resulting curve shapes. In particular, for the problem of curve reconstruction from a smooth physical shape, specifying the precise segment lengths significantly improves the accuracy of the reconstruction - especially in the case of sparse sampling.

The schemes presented herein constitute preliminary steps toward solving the Morphosense shape reconstruction problem, introduced in Section 1. They incorporate the important feature of reproducing the precise curvilinear distances between sensor locations, but the method of Section 5 interpolates parametric derivative vectors, rather than Serret-Frenet frames with unit vectors (the 
choice of derivative magnitudes was briefly discussed in Section 5.3). $C^{2} \mathrm{PH}$ quintic splines cannot interpolate Serret-Frenet frames, but one may come closer to this requirement by relaxing from $C^{2}$ to $G^{2}$ continuity. Further investigations along these lines will be reported in due course.

\section{References}

[1] G. Albrecht and R. T. Farouki (1996), Construction of $C^{2}$ Pythagorean-hodograph interpolating splines by the homotopy method, Adv. Comp. Math. 5, 417-442.

[2] H. I. Choi, D. S. Lee, and H. P. Moon (2002), Clifford algebra, spin representation, and rational parameterization of curves and surfaces, Adv. Comp. Math. 17, 5-48.

[3] D. David and N. Sprynski (2006), Method and device for acquisition of a geometric shape, Patent No. wo/2006/095109.

[4] G. Farin (2002), Curves and Surfaces for Computer Aided Geometric Design, 5th edition, Academic Press, San Diego.

[5] R. T. Farouki (2008), Pythagorean-Hodograph Curves: Algebra and Geometry Inseparable, Springer, Berlin.

[6] R. T. Farouki, M. al-Kandari, and T. Sakkalis (2002), Structural invariance of spatial Pythagorean hodographs, Comput. Aided Geom. Design 19, 395-407.

[7] R. T. Farouki, M. al-Kandari, and T. Sakkalis (2002), Hermite interpolation by rotation-invariant spatial Pythagorean-hodograph curves, Adv. Comp. Math. 17, 369-383.

[8] R. T. Farouki, B. K. Kuspa, C. Manni, and A. Sestini (2001), Efficient solution of the complex quadratic tridiagonal system for $C^{2} \mathrm{PH}$ quintic splines, Numer. Algor. 27, 35-60.

[9] R. T. Farouki, C. Manni, and A. Sestini (2003), Spatial $C^{2}$ PH quintic splines, in Curve and Surface Design: Saint-Malo 2002 (T. Lyche, M.-L. Mazure, and L. L. Schumaker, eds.), Nashboro Press, 147-156.

[10] R. T. Farouki, C. Manni, F. Pelosi, and M. L. Sampoli (2012), Design of $C^{2}$ spatial Pythagorean-hodograph quintic splines by control polygons, in (J. D. Boissonnat et al., eds.), Lecture Notes in Computer Science Vol. 6920, 253-269, Springer.

[11] M. Huard (2011), Serret-Frenet frame interpolation under length constraints for surface reconstruction, Actes des journées du GTMG.

[12] M. Huard, N. Sprynski, N. Szafran, and L. Biard (2012), Reconstruction of quasi developable surfaces from ribbon curves, Numer. Algor. to appear.

[13] F. Pelosi, M. L. Sampoli, R. T. Farouki, and C. Manni (2007), A control polygon scheme for design of planar $C^{2} \mathrm{PH}$ quintic spline curves, Comput. Aided Geom. Design 24, 28-52.

[14] N. Sprynski (2007), Reconstruction de courbes et surfaces à partir de données tangentielles, $\mathrm{PhD}$ thesis, Université Joseph Fourier, Grenoble. 